\title{
Incidencia de diabetes gestacional y su relación con obesidad en embarazadas chilenas
}

\author{
Andrea Huidobro $\mathrm{M}^{1}$, Anthony Fulford ${ }^{2 a}$, Elena Carrasco $\mathrm{P}^{3 \mathrm{~b}}$. \\ Incidence of gestational diabetes \\ and relationship to obesity \\ in Chilean pregnant women
}

Background: Gestational diabetes (GDM) is associated to a worse outcome of pregnancy. This justifies efforts for finding possible causes of GDM that would allow implementing preventive interventions. Aim: To study incidence of GDM and its relation with obesity and other traditional risk factors. Material and methods: A retrospective study was performed in 234 women who had delivered a singleton during the last 12 months, attended in an outpatient clinic in Santiago, Chile. Familiar and personal history, body mass index (BMI), obstetrical-related pathology and data about the labor and the newborn were analyzed. Results: GDM was diagnosed in $11.2 \%$ of the women. BMI before pregnancy was $26.6 \pm 4.4 \mathrm{~kg} / \mathrm{m} 2$ (mean $\pm \mathrm{SD}$ ) and it was 25 or over in $37.8 \%$ of women. Women who developed GDM had significantly higher $\mathrm{BMI}$ in the pre-pregnancy stage and in the second and third trimester of pregnancy ( $\mathrm{p}<0.001$ ). The average age was greater in the GDM group ( $31 \pm 0.2$ yr versus $26 \pm 0.41 \mathrm{yr}$ ). Incidence of GDM was $14.4 \%$ among women 25 years old or older and increased to $21.4 \%$ when they had, in addition, a BMI of 25 or over. Age, BMI, and family history of diabetes were all independently correlated with the development of GDM. Elective caesarean sections were more common in GDM than in non-GDM women $(p=\measuredangle 0.01)$ and complications were present in $3 / 23$ of newborns of women with GDM and 2/199 among women without GDM ( $p<0.01)$ Conclusions: GDM and obesity are highly prevalent in Chilean pregnant women. BMI, first degree relative with DM and age are independent risk factors for the development of GDM (Rev Méd Chile 2004; 132: 931-8).

(Key Words: Body mass index; Diabetes, gestational; Obesity)

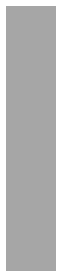

\footnotetext{
Recibido el 11 de dicembre, 2003. Aceptado en versión corregida el 18 de mayo, 2004. Trabajo financiado por el Wellcome Trust.

${ }^{1}$ Unidad de Asistencia Nutricional Intensiva, Hospital Regional de Talca, Chile. ${ }^{2} \mathrm{MRC}$ International Group, Public Health Nutrition Unit, Department of Epidemiology and Population Health, London School of Hygiene and Tropical Medicine, UK; ${ }^{3}$ Clínica de Diabetes, Hospital San Juan de Dios, Universidad de Chile, Santiago de Chile.

aEstadístico, $\mathrm{PhD}$.

${ }^{b}$ Nutricionista, MSc.
}

Correspondencia a: Dra. Andrea Huidobro M. Unidad de Asistencia Nutricional Intensiva, Hospital Regional de Talca. 1 Norte 1990, Talca. Fono: 71-209411.

E-mail: andrea.huidobro@lshtm.ac.uk 
T a Diabetes Mellitus (DM) es una enfermedad Lde alta prevalencia, que se relaciona fuertemente a la vida sedentaria y a una dieta occidental. Estadísticas de la Organización Mundial de la Salud (OMS) y proyecciones indican que los niveles crecientes de obesidad a nivel mundial conducirán a un incremento desmesurado en la prevalencia de diabetes Mellitus tipo $2^{1}$. La epidemia se cuadruplicaría en países en desarrollo ${ }^{2}$ y la morbilidad y mortalidad aumentaría 6 veces $^{3}$. En la población general chilena, se ha comunicado un aumento de $1,2 \%$ a $6,5 \%$ en la prevalencia de DM en los últimos años.

La Diabetes Gestacional (DG) se define como intolerancia a la glucosa que se reconoce por primera vez durante el embarazo ${ }^{4}$ y se ha postulado como una variante de DM tipo 2. Su prevalencia varía ampliamente, dependiendo de la población en estudio y los criterios diagnósticos utilizados ${ }^{5}$, llegando a 6,3\% cuando se diagnostica con el test de tolerancia con $75 \mathrm{~g}$ de glucosa (75 g 2-h TTOG) ${ }^{6}$. Chile tiene una alta prevalencia de diabetes, sin embargo no hay publicaciones recientes respecto a DG. El estudio de Mella y cols, entre 1982-84, documentó una incidencia de 13,8\% de DG en mujeres consideradas en riesgo ${ }^{7}$.

Ante la falta de datos recientes en la prevalencia de obesidad en embarazo (uno de los factores de riesgo más importantes para el desarrollo de DG) y de incidencia de DG en nuestra población, se propuso realizar un estudio retrospectivo para estimarlas.

\section{MÉTodos}

Se realizó un estudio retrospectivo en 234 mujeres que tuvieron un hijo único durante los doce meses anteriores al estudio. Las pacientes fueron seleccionadas a partir de las fichas médicas de aquellas que se controlaron en el policlínico Andes durante su embarazo y tuvieron su parto entre abril de 2000 y abril de 2001. Cuatro pacientes que presentaron aborto durante el primer trimestre y dos mujeres con embarazos gemelares fueron excluidas del estudio.

El estudio fue aprobado por las Comisiones Éticas de la London School of Hygiene and Tropical Medicine, University of London y del Hospital San Juan de Dios, Universidad de Chile.
Se recolectaron los datos personales, socioeconómicos, obstétricos y de historia familiar de diabetes e hipertensión arterial. Se registró también el índice de masa corporal (IMC) previo al embarazo, entre las semanas 24 y 28 y entre las semanas 32 y 36 del embarazo. Se buscó la presencia o ausencia de DG, hipertensión, preeclampsia o colestasia durante el embarazo y se recolectaron los datos del tipo de parto, peso y condición del recién nacido.

Sólo las pacientes con dos glicemias basales iguales o superiores a $105 \mathrm{mg} / \mathrm{dL}$ o con una glicemia posterior a carga oral de $75 \mathrm{~g}$ de glucosa (TTOG) igual o superior a $140 \mathrm{mg} / \mathrm{dL}$ se consideraron con diabetes gestacional. Esta clasificación se hizo de acuerdo a las normas ministeriales que se basan en las de la Asociación Americana de Diabetes (ADA). Toda paciente tenía un primer TTOG entre las semanas 24 y 28. Si éste no era posible o si existía sospecha clínica de diabetes gestacional a pesar de un primer examen normal, se realizaba un segundo TTOG entre las semanas 32 a 36 .

Estadísticas: Las asociaciones entre DG y cada una de las variables en estudio se analizaron en forma individual utilizando el test exacto de Fisher para las variables categóricas y ANOVA para las continuas. Las variables predictoras consideradas potencialmente causales, tales como edad, número de embarazos previos, IMC e historia familiar de DM tipo 2, se analizaron posteriormente en un modelo de regresión logística. Nuestros hallazgos fueron finalmente comparados con los datos del estudio chileno realizado en $1982^{8}$.

\section{Resultados}

Doscientas treinta y cuatro mujeres fueron seleccionadas para este estudio. La edad promedio fue de 26,6 años, con un rango entre 15 y 41.

Las mujeres de la muestra eran predominantemente caucásicas (87,6\%), el resto comprendió 11 mujeres $(4,7 \%)$ con ambos padres de origen mapuche y $18(7,7 \%)$ con un padre mapuche y uno caucásico.

La mayoría de las mujeres estaban casadas $(40,8 \%)$ o viviendo con sus parejas $(34,7 \%)$, mientras que $1,3 \%$ estaban separadas y $23,6 \%$ carecían de una pareja estable. El nivel de educa- 
ción de las pacientes estudiadas se muestra en la Tabla 1.

La paridad promedio fue $1,3 \pm 1,7$ hijos por paciente, con un rango entre 0 y 12. Los datos sobre paridad, abortos y características de partos anteriores se muestran en la Tabla 2.

El promedio de edad gestacional al primer control fue de 14,5 semanas (4-38 semanas). Ochenta y uno coma cuatro por ciento de las

\section{Tabla 1. Nivel educacional en 234 mujeres en control de embarazo en el Policlínico Andes entre abril de 2000 y abril de 2001}

\begin{tabular}{|lc|}
\hline Nivel de estudios & Porcentaje (\%) \\
\hline Analfabetas & 0,9 \\
Enseñanza Básica & 22,3 \\
Enseñanza Media & 59,7 \\
Estudios superiores & 16,7 \\
\hline
\end{tabular}

pacientes atendieron a su primer control antes de la semana 20 de embarazo.

El IMC previo al embarazo (dato recolectado anamnésicamente) fue $26,6 \pm 4,4 \mathrm{~kg} / \mathrm{m}^{2}$ (promedio \pm desviación estándar), con rangos entre 15,9 y 48,6. El IMC fue menor a 20 en $7,7 \%$ de las mujeres, normal en $54,5 \%$ y excedido en $37,8 \%$ de ellas.

Ninguna de las mujeres tenía el diagnóstico de diabetes previo a su embarazo. Los exámenes diagnósticos se realizaron en forma oportuna en 223 pacientes (95,3\%). Se diagnosticó DG en 25 de estas mujeres $(11,2 \%)$. Niveles elevados de glicemia basal diagnosticaron DG en sólo una paciente. Diecisiete de las pacientes se diagnosticaron con el primer TTOG y otras siete se diagnosticaron con el segundo. Cinco de las mujeres diagnosticadas entre las semanas 32 y 36 tenían un TTOG previo normal, las dos restantes no tenían el primer examen realizado.

No se encontró diferencias en la historia obstétrica de las pacientes que presentaron DG y las que no la presentaron, excepto que mujeres

Tabla 2. D atos de embarazos previos en mujeres con y sin diabetes gestacional

\begin{tabular}{|lrrrrc|}
\hline & Total & DG & \multicolumn{2}{c|}{$\begin{array}{c}\text { Test de Fisher } \\
\text { Valor P }\end{array}$} \\
& & $\mathrm{n}$ & $\mathrm{n}$ & $(\%)$ & \\
\hline Número de hijos & 0 & 90 & 7 & 7,8 & 0,23 \\
& 1 a 3 & 136 & 14 & 10,3 & $0,03^{*}$ \\
Abortos & $>3$ & 17 & 3 & 17,6 & 0,27 \\
& 0 & 195 & 20 & 10,3 & 0,6 \\
Macrosomía & 1 & 25 & 2 & 8,0 & 0,5 \\
RCIU & $>1$ & 14 & 2 & 14,3 & 0,45 \\
Malformaciones & Presente & 10 & 3 & 30,0 & 0,07 \\
Mortinato & Presente & 8 & 1 & 12,5 & 0,58 \\
Preeclampsia & Presente & 1 & 1 & 100,0 & 0,1 \\
Colestasia & Presente & 5 & 2 & 40,0 & 0,09 \\
& Presente & 9 & 3 & 33,3 & 0,05 \\
\hline
\end{tabular}

*Diferencia significativa

RCIU: Retardo del crecimiento intrauterino. 
con entre uno y tres embarazos presentaron DG con mayor frecuencia. Sin embargo, el antecedente de preeclampsia y de macrosomías no alcanzó significación estadística, tal como se muestra en la Tabla 2.

Se definió madres en riesgo de presentar DG a las que tenían historia de RCIU, abortos previos, malformaciones o mortinatos en embarazos anteriores. Individualmente, ninguna de estas condiciones estuvo asociada a la presencia de DG (Tabla 2). El grupo de las madres en riesgo, así definidas, presentó mayor incidencia de DG que el resto, alcanzando 17,4\% (4 de 23). Esta diferencia sin embargo, no alcanzó significancia estadística.

Los factores de riesgo que fueron diferentes entre mujeres que desarrollaron DG y las que no, se muestran en la Tabla 3. El IMC fue significativamente mayor en las mujeres que desarrollaron DG, tanto en la medición basal $(27,7 \pm 5$ vs $24,2 \pm 4,2)$, como entre las semanas 24 a $28(31,7 \pm 5$ vs $27,9 \pm 4,4, \mathrm{p}=0,0006)$ y 32 a $36(33,5 \pm 5,1$ vs $29,6 \pm 4,7, p=0,001$ ).

La prevalencia de DG en mujeres mayores de 25 años fue $14,4 \%$ y si además presentaban un IMC de 25 o más, la incidencia se elevaba a 21,4\%.

El antecedente familiar de hipertensión 0 diabetes estaba asociado a la presencia de DG. Por otro lado, las pacientes que presentaron DG tuvieron mayor prevalencia de hipertensión (8,3\% vs $6,5 \%)$, sin embargo, esta diferencia no alcanzó significancia estadística $(\mathrm{p}>0,05)$.
En el modelo de regresión múltiple el IMC, antecedente familiar de diabetes y edad resultaron independientemente asociados al desarrollo de DG. El IMC y edad estaban relacionados entre sí, sin embargo la sobreposición entre ambos da cuenta de sólo una fracción del efecto de cada uno. Aunque el número de embarazos previos presentó asociación con el desarrollo de DG, este efecto no fue independiente de la edad. Los odds ratios o razones de disparidad de estas variables se muestran en la Tabla 4.

La comparación en algunas de las características de los partos entre mujeres con DG y el resto, se resume en la Tabla 5. Ciento setenta y dos de los partos (73,8\%) fueron vaginales, $19,7 \%$ fueron cesáreas electivas y 6,4\% fueron cesáreas de urgencia. Las cesáreas electivas fueron más frecuentes en las pacientes con DG (50\% vs $17,1 \%$, $\mathrm{p}=0,00038$ ). Aunque las mujeres con DG presentaron mayor frecuencia de cesáreas de urgencia, esta diferencia no alcanzó significancia estadística $(8,3 \%$ vs $6,0 \%, p=0,9)$.

Cinco recién nacidos presentaron complicaciones (distress respiratorio, hipoglicemia, prematurez, mortinato y muerte neonatal). Las complicaciones estuvieron presentes en 3/23 (13\%) de los partos de mujeres con DG y en 2/199 (1\%) del resto de las pacientes $(p=0,00888)$.

El peso promedio de los recién nacidos fue $3.336 \pm 577 \mathrm{~g}(740-4.600 \mathrm{~g})$ y la talla promedio de $49,3 \pm 3,1 \mathrm{~cm}$. Ninguna de estas medidas se relacionó a la presencia de DG, al nivel de glicemia basal 0 al resultado de los TTOG.

Tabla 3. D iferencias en factores de riesgo entre pacientes con y $\sin D$ G

\begin{tabular}{|llll|}
\hline & DG & Tolerancia normal & Valor P \\
\hline Edad & $31,9 \pm 6,8$ & $26 \pm 5,9$ & $0,00005^{*}$ \\
IMC basal & $27,7 \pm 5$ & $24,2 \pm 4,2$ & $0,0006^{*}$ \\
Glicemia basal & $92,1 \pm 29 \mathrm{mg} / \mathrm{dL}$ & $80,26 \pm 8 \mathrm{mg} / \mathrm{dL}$ & $0,0001^{*}$ \\
Familiar con DM & $30,4 \%$ & $8,5 \%$ & $0,005^{*}$ \\
Familiar con HTA & $47,8 \%$ & $27,6 \%$ & $0,04^{*}$ \\
\hline
\end{tabular}

HT: Hipertensión arterial

${ }^{*}$ Diferencia significativa

Valores expresados en promedio más desviación estándar. 
Tabla 4. Análisis de regresión logística

\begin{tabular}{|lccccc|}
\hline Variable & OR & Error St. & Z & Sig. & $\begin{array}{c}95 \% \\
\text { Interval. Conf }\end{array}$ \\
\hline Edad & 1,17 & 0,056 & 3.287 & 0,001 & $1.065-1.285$ \\
IMC & 1,13 & 0,056 & 2.532 & 0,011 & $1.029-1.247$ \\
Familia con DM & 3,47 & 2,084 & 2.073 & 0,038 & $1.070-11.257$ \\
Log Emb Prev & 0,47 & 0,226 & -1.569 & 0,117 & $0,182-1.208$ \\
\hline
\end{tabular}

Log Emb Prev: Log del número de embarazos previos.

OR: Odds Ratio

Tabla 5. D iferencias en las características del parto entre pacientes con y sin D G

\begin{tabular}{|lccl|}
\hline & DG & Tolerancia normal & Valor P \\
\hline Edad gestacional (semanas) & $38,6 \pm 1,3$ & $38,9 \pm 1,8$ & 0,4 \\
Parto vaginal (\%) & 41,7 & 76,9 & $0,0002^{*}$ \\
Cesárea (\%) & 50 & 17,1 & $0,0004^{*}$ \\
Cesárea urgencia (\%) & 8,3 & 6,0 & 0,9 \\
Peso recién nacido (g) & $3.385,8$ & $3.354 \pm 533$ & 0,6 \\
Talla recién nacido (cm) & $48,6 \pm 4,3$ & $49,5 \pm 2,7$ & 0,3 \\
Complicaciones (\%) & 12,5 & 1 & $0,009^{*}$ \\
\hline
\end{tabular}

${ }^{*}$ Diferencia estadísticamente significativa.

\section{Discusión}

Este estudio sugiere que las mujeres embarazadas chilenas de nivel socio económico medio-bajo presentan una alta incidencia de diabetes gestacional. Tienen también una alta prevalencia de sobrepeso y obesidad en la etapa pregestacional, que llega a $37,8 \%$ y en las mayores de 25 años, a $47,8 \%$ (este dato no se muestra), definido según criterio de la $\mathrm{OMS}^{1}$.

El IMC estaría asociado al desarrollo de DG, de la misma forma en que se asocia a la presencia de DM tipo 2 en la población general. Este hecho refuerza la posición de algunos autores que definen a la DG como una variante de la DM tipo 2.
La frecuencia levemente elevada, aunque no significativa, de hipertensión en las mujeres con DG concuerda con el estado de insulino resistencia gatillado durante el embarazo y apoya la idea de DG como modelo de DM tipo 2.

Las mujeres con DG presentaron una tendencia, aunque no significativa, a tener mayor frecuencia de una historia obstétrica adversa. El antecedente de macrosomía da cuenta de la mayoría de esta diferencia. Una muestra insuficiente podría explicar la falta de significación estadística.

Edad, IMC y el antecedente de un pariente en primer grado con diabetes demostraron ser factores independientes asociados a DG de acuerdo a este estudio. El sobrepeso ha sido ampliamente 
discutido como un reconocido factor de riesgo para diabetes. El antecedente de un pariente con diabetes traduce la importancia de la interacción herencia y ambiente en el desarrollo de DG en la misma forma en que se asocia a la DM tipo 2.

La relación directa de la edad con el desarrollo de DG puede reflejar la existencia de otros factores de riesgo no analizados en este estudio, que requieren tiempo para expresarse y desarrollar insulino resistencia. Es posible que algunos marcadores sanguíneos actúen de esta manera. En este sentido, el perfil de ácidos grasos plasmáticos, específicamente la razón w-3/w $6^{9-11}$ y los marcadores de inflamación derivados de los adipocitos $^{12-14}$ han demostrado estar asociados a la insulino resistencia. Estudios fisiológicos, así como de intervención con suplementos de aceite de pescado, sugieren la posibilidad que la razón w-3/ w-6 podría explicar el desarrollo de DM tipo $2^{15-17}$. Conociendo el rol de los ácidos grasos en la secreción de citoquinas derivadas de los adipocitos ${ }^{18,19}$, los ácidos grasos podrían gatillar la insulino resistencia per se 0 a través de la acción de estas citoquinas.

La posible existencia de factores de riesgo aún desconocidos, relacionados a la patogenia de DG, apoya la política actual de evaluar a toda mujer embarazada para descartar o diagnosticar DG y no sólo a las portadoras de factores de riesgo tradicionales. Estudios recientes apoyan esta posición. Es así como Griffin y cols, en un estudio prospectivo randomizado mostraron que el screening universal detecta más casos de DG, permite un diagnóstico más temprano y está asociado a una mayor probabilidad de término exitoso del embarazo que un screening basado en factores de riesgo 20 .

El mayor riesgo de complicaciones para los recién nacidos de mujeres con DG en este estudio, es especialmente llamativo. Los recién nacidos de mujeres sin DG presentaron similar frecuencia de complicaciones que la descrita para la población general, mientras que los hijos de mujeres con DG presentaron diez veces más complicaciones. Este hecho fortalece la importancia de realizar un diagnóstico precoz y un tratamiento oportuno de la DG, lo cual podría prevenir estas complicaciones. En este mismo sentido, el 75 g-2 h TTOG, siendo más sensible que el 100 g-3 h TTOG, constituiría un buen instrumento para discriminar eficientemente a una población en alto riesgo de presentar complicaciones neonatales.

El mayor riesgo de cesárea en las mujeres con DG se repite en este estudio, aunque de manera más marcada que en otras publicaciones ${ }^{21,22}$. Debido a que el equipo médico estaba en antecedente del diagnóstico de DG, es posible que tuvieran menores exigencias para indicar un parto quirúrgico. Sin embargo, no es posible discutir más profundamente esta materia debido a que carecemos de la información médica que justificó la decisión del tipo de parto.

Este estudio concuerda con los resultados obtenidos por Mella y cols ${ }^{7}$. Clasificando a las pacientes bajo los mismos criterios de riesgo utilizados por Mella, nuestro estudio arrojó una prevalencia de 19,6\% de DG en mujeres en riesgo $\mathrm{y}$ de $3,3 \%$ en las mujeres sin factores de riesgo (estos datos no se muestran). Llama la atención el aumento de prevalencia de DG en ambos grupos entre este estudio y el de 1982. Sin embargo, esto puede ser explicado por la diferencia en el test diagnóstico utilizado, ya que el 3-h $100 \mathrm{~g}$ TTOG con cuatro mediciones de glicemia es un método diagnóstico más estricto y puede no diagnosticar pacientes con DG según el 2 h-75 g TTOG.

Las poblaciones de ambos estudios son similares en edad y prevalencia de obesidad, sin embargo difieren en otras características. A pesar de presentar con menor frecuencia malos antecedentes obstétricos y antecedentes familiares de DM tipo 2, nuestra población presenta una mayor prevalencia de DG que la población estudiada en 1982.

En conclusión la DG y la obesidad son altamente prevalentes en mujeres embarazadas chilenas. El IMC, antecedentes familiares de DM tipo 2 y edad han demostrado ser factores independientes de riesgo para esta condición. Por otro lado, la DG está asociada a un mayor riesgo de cesárea y mayores complicaciones para el recién nacido.

Los resultados de este estudio debieran conducir a nuevos estudios destinados a establecer relaciones en el tiempo entre obesidad y el desarrollo de insulino resistencia y DG, así como la relación entre estas condiciones y el desarrollo de hipertensión inducida por el embarazo. Debido 
a que la evidencia sugiere que factores de riesgo distintos a los tradicionalmente estudiados podrían ser responsables del desarrollo de insulino resistencia, los autores proponen futuros estudios

\section{REFERENCIAS}

1. WHO: Obesity. Preventing and managing the global epidemic. Geneva: WHO; 1998 3-5th June 1997. WHO/NUT/NCD/98.1, 1998.

2. King H, Aubert R, Herman W. Global Burden of Diabetes, 1995-2025. Prevalence, numerical estimates and projections. Diabetic Care 1994; 17: 961-9.

3. Mc Larty D, Unwin N, Kitange H, Alberti K. Diabetes Mellitus as a cause of death in SubSaharan Africa: Results of a Community-based study in Tanzania. Diabetic Medicine 1996; 13: 990-5.

4. National Diabetes Data Group: Classification and Diagnosis of Diabetes Mellitus and other categories of glucose intolerance. Diabetes 1979; 28: 1039-44.

5. Peruchini D, Fischer U, Spinas G, Huch R, Huch A, LECHMAN R. Using fasting plasma glucose concentrations to screen for gestational diabetes mellitus: prospective population based study. BJM 1999; 319: 812-5.

6. Moses R, Moses M, Russell K, Schier G. The $75 \mathrm{~g}$ Glucose Tolerance Test in Pregnancy. Diabetes Care 1998; 21: 1807-11.

7. Melia I, López G, Durruty P, García de los Ríos M. Frecuencia de diabetes gestacional en embarazadas en riesgo diabético de Santiago, Chile. Bol Of Sanit Panam 1990; 109: 342-9.

8. Mela I, López G, Durruty P, García de los Ríos M. Prevalencia de diabetes gestacional en mujeres en riesgo en Santiago, Chile. Rev Méd Chile 1981; 109: 869-75.

9. Ginsberg B, Brown T, Simon I, Spector A. Effect of the membrane lipid enviroment on the properties of insulin receptors. Diabetes 1981; 30: 773-80.

10. Borkman M, StorLien L, Pan D. The relationship between insulin sensitivity and the fatty acid prospectivos para determinar una posible relación de ciertos marcadores sanguíneos y el desarrollo de DG.

composition of skeletal muscle phospholipids. N Engl J Med 1993; 328: 238-44.

11. Pan D, Lu山OJa S, Milner M. Skeletal muscle membrane lipid composition is related to adiposity and insulin action. J Clin Invest 1995; 96: 2802-8.

12. Nilsson J, Jovinge S, Niemman A, Reneiand R, Lithell $H$. Relation between plasma tumor necrosis factor alpha and insulin sensitivity in elderly men with non insulin dependent diabetes mellitus. Arterioesc Thromb 1998; 18: 1199-202.

13. Hotamisligil G, Shargill N, Sieggelman B. Adipose expression of tumor necrosis factor alpha. Direct role in obesity-linked insulin resistance. Science 1993; 259: 87-90.

14. Pickup J, Mattock M, Chusney G. NIDDM as a disease of the innate immune system: association of accute phase reactants and interleukine- 6 with metabolic syndrome X. Diabetologia 1997; 40: 1286-92.

15. Mori Y, MuraKawa Y, Yokohama J, Tajima M, Ikeda $\mathrm{Y}$, Nobukata $\mathrm{H}$ et al. Effect of highly purified eicosapentaenoic acid ethil ester on insulin resistance and hypertension in Dahl salt sensitive rats. Metabolism 1999; 48: 1089-95.

16. Storlen L, Jenkins A, Chisholm D, Pascoe W, Khouri $\mathrm{S}$, KRAEGEN E. Influence of dietary fat composition on development of insulin resistance in rats. Relationship to muscle triglyceride and $n-3$ fatty acids in muscle phospholipids. Diabetes 1991; 40: 280-9.

17. Pelukanova T, Kohonut M, Valek J, Base J, Kazdova L Insulin secretion and insulin action related to the serum phospholipid fatty acid pattern in healthy men. Metabolism 1989; 38: 88-92.

18. Wachtier P, Konig W, Senkal M, Koler M. Influence of a total parenteral nutrition enriched with $n-3$ fatty acids on leukotriene synthesis of periferal leukocites and systemic cytokine levels in patients with major surgery. J Traum 1997; 42: 191-8. 
19. Purasiri P, MurRay A, Richardson S. Modulation of cytokine production in vivo by dietary essencial fatty acids in patients with colorectal cancer. Clin Sci 1994; 87: 711-7.

20. Griffin ME, Coffey M, Johnsont H, Scanlon P, Foley M, Stronget J ET aL. Universal vs risk factor-based screening for gestational diabetes mellitus: detection rates, gestation at diagnosis and outcome. Diabet Med 2000; 17: 26-32.
21. Jensen DM, Sorensen B, Feilberg-Jorgensen N, WesTERGAARD JG, BECK-NIEISEN H. Maternal and perinatal outcomes in 143 Danish women with gestational diabetes mellitus and 143 controls with a similar risk profile. Diabet Med 2000; 17: 281-6.

22. Moses RG, Knights SJ, Lucas EM, Moses M, Russell KG, Coleman KJ et al. Gestational Diabetes: Is a higher cesarean section rate inevitable? Diab Care 2000; 23: 15-7.

\section{Agradecimientos}

Los autores expresan sus más sinceros agradecimientos a las autoridades, personal administrativo y de maternidad del policlínico Andes de Quita Normal, especialmente a la matrona Lorena Villanova por su interés en hacer este estudio expedito. 